$$
1995119511
$$

N95- 25931

44644

APPENDIX 8

Cosmic Ray Particles with Different LET Values Under various Thicknesses of shielding In Low Altitude Orbits: Calculations and Cosmos-2014 Measurements

by

A.M. Marenny, R.A. Nymmik, A.A. Suslov, E.V. Benton, A.I. Frank and E.R. Benton

(210) 


\title{
COSMIC RAY PARTICLES WITH DIFFERENT LET VALUES UNDER VARIOUS THICKNESSES OF SHIELDING IN LOW ALTITUDE ORBITS: CALCULATIONS AND COSMOS-2044 MEASUREMENTS
}

\author{
A. M. Marenny, R. A. Nymax, A. A. Suslov, † E. V. Benton $\ddagger$ A. L. Frant \\ and E. R. Bentont \\ - Research Center for Spacecraft Radiation Sajety, Moscow 123182, U.S.S.R.; Hinstitute of Nuclear \\ Physics, Moscow Stace University, Moscow 119989, U.S.S.R.; †University of San Francisco, \\ San Francisco, CA 94117 , U.S.A.
}

(Received 24 June 1991; in revised form 9 July 1991)

\begin{abstract}
Fluxes of cosmic ray particles with different LET values were measured on board the Cosmos-2044 biosatellite under various thicknesses of shielding by stacks of CR-39 and nitrocellulose plastic nuclear track detectors (mounted outside the satellite). The component composition of the particles detected under shieldings of $0.1-2.5 \mathrm{~g} \mathrm{~cm}^{-2}$ is verified by comparing experimental data with the results of model simulations of the fluxes of galactic cosmic ray particles and of radiation belt protons.
\end{abstract}

\section{INTRODUCTION}

THE RADUATION fields in the Earth's vicinity are generated by the particles which are commonly grouped, according to some criteria (mainly their origin, composition and energy) into certain components, namely, galactic and solar cosmic rays (GCR and $S C R$ ), radiation belt $(R B)$ particies and anomalous fluxes (AF). To date, numerous experiments have been carried out on board Soviet and U.S. spacecraft, yielding copious data on the fluxes and spectra of protons and heavy ions in the Earth's vicinity (see, for example, Benton and Parnell, 1988; Marenny et al., 1987). Almost all of the data have been obtained using solid-state nuclear track detectors (SSNTD) (Marenny, 1987) which are nocable for their essential characteristic of a detection threshold expressed by the smallest LET value at which detection is possible.

In the present work, an attempt is made to analyze the components of SSNTD-detected particle fluxes under various thicknesses of shielding. The experimental data are supplemented with simulations calculated by models of the radiation environment. Only $G C R$ and $R B$ particles are included because other heavy cosmic ray components did not penetrate to greater shielding thicknesses than $0.05 \mathrm{~g} \mathrm{~cm}^{-2}$ during the Cosmos-2044 tight.

\section{EXPERIMENT}

The Cosmos-2044 mission lasted for 13.8 days from 15 to 29 September 1989 . The biosateilite orbit was elliptical, with a perigee and apogee of 216 and $294 \mathrm{~km}$, respectively, and an inclination of $82.3^{\circ}$. Four flat, lidded containers holding a variety of dosimetric equipment were mounted outside the satellite.

The experimental results presented bere were obtained by processing three SSNTD stacks, one at IBMP (Moscow) and two at USF (San Francisco). The IBMP stack of $90 \times 50 \times 16 \mathrm{~mm}$ dimensions contained 20 Soviet-made KNC-type nitrocellulose detectors of $800 \mu \mathrm{m}$ thickness each. The detectors were erched in a $6 \mathrm{~N} \mathrm{NaOH}$ solution for $5 \mathrm{~b}$ at $50^{\circ} \mathrm{C}$. The tracks were scanned with a stereo-microscope at $80 \times$ magnification. The penetrating (a cylinder or two cones) or single-cone (of at least $100 \mu \mathrm{m}$ length) tracks were selected by counting, and correspond with the GCR particles of $Z \geqslant 6$ and LET $\geqslant 1600$ $\left(\mathrm{MeV} \mathrm{mm}^{-}\right) \mathrm{g}^{-1}$ (under the given etching conditions).

The USF stacks were of $3 \mathrm{~cm}$ diameter and included CR-39 and Cronar polyester layers. The CR. 39 SSNTDs were processed in a $6.25 \mathrm{~N} \mathrm{NaOH}$ solution for 7 days at $50^{\circ} \mathrm{C}$. The bulk etch, $B$, was measured for each of the detectors. Pairs of detectors were reassembled in their flight orientations and the two adjacent inner surfaces were scanned with an optical microscope. This procedure permitted the particles to be separated into short-range (SR) (matching tracks appeas on two inner surfaces only) and long-range (LR) (matching tracks appear on all four surfaces of the detector pairs) GCR particle tracks. The SR particles include the secondary SR particles from target nuclei within the plastic and the stopping primary GCR and trapped particles. All

+USF porion of the work partially supported by NASA Grant Nos NCC2-521 (NASA-Ames Research Center) and NÁG9-23S (NASA-Johnson Space Center). 


\section{A. M. MARENNY el al.}

protons were detected as $S R$ particles because of their short registration ranges in CR-39. The GCR particles include the primary GCRs and the LR $Z \geqslant 2$ secondaries which are mainly the GCR projectile fragments. The $Z \geqslant 2$ stopping GCR particles can also contribute to the SR tracks, but with a low probability.

\section{ANALYTICAL PROCEDURE}

\subsection{Galactic cosmic rays}

The GCR particle fluxes outside the Earth's mag. netosphere were calculated in terms of the model proposed by Suslov and Nymmik (1988) and described in detail by Suslov and Nymmik (1990). In the model, the energy spectra $F_{i}(E, t)$ of the $1 \leqslant Z \leqslant 28$ GCR particles are inferred from the particle rigidity spectra $\Phi_{i}(R, t)$ :

$$
F_{i}(E, t) \mathrm{d} E=\Phi_{i}(R, t) \frac{A_{t} \beta}{|z|} \mathrm{d} R,
$$

where $A_{j}$ is the atomic number of a nucleus of species $i ; R_{i}$ is particle rigidity; $t$ is time.

The rigidity spectra are defined by the phenomenological model

$$
\Phi_{i}(R, t)=\frac{D_{i} \beta^{a_{i}}}{R_{i}^{\prime}}\left(\frac{R}{R+R_{0}(t)}\right)^{\Delta_{i}(t)},
$$

where $D_{i}, \alpha_{i}$ and $y_{i}$ are the constants which characterize the spectra of particles of certain species whose values were determined through the available set of experimental data on the GCR particle fluxes during the previous solar cycles.

The data used to calculate the fluxes of all particles can be found in Standards for Galactic Cosmic Rays (1991); the constants used in calculating the energy spectra of the basic elements are presented in Table 1. The value of $R_{0}$ is defined by the solar activity level and by the delay of the $C R$ particle flux variations relative to Wolf numbers. During the experiment, $R_{0}$ proved to be $0.88 \mathrm{GV}$.

The power-law exponent in (2) is determined by the formula

$$
\begin{aligned}
\Delta_{1}(t)=5.5\left[1+a_{i} \exp \left(\frac{z_{i} \beta R}{A_{1} b_{i}}\right)\right] \\
+1.0 \operatorname{sgn}\left(z_{1}\right) \frac{\beta R}{R_{0}} \exp \left(-\frac{\beta R}{R_{0}}\right) .
\end{aligned}
$$

Table 1. Values of constants used in ealculating energy

\begin{tabular}{lrrrrr}
\multicolumn{7}{c}{ spectra } \\
\hline Element & $Z_{1}$ & $A_{1}$ & $D_{i}$ & $a_{i}$ & $\gamma_{i}$ \\
\hline $\mathrm{H}$ & 1 & 1 & $2.0 \mathrm{E} 04$ & 3.0 & 2.75 \\
$\mathrm{He}$ & 2 & 4 & $3.5 \mathrm{E} 03$ & 3.0 & 2.75 \\
$\mathrm{C}$ & 6 & 12 & $9.6 \mathrm{E} 01$ & 3.1 & 2.75 \\
$\mathrm{O}$ & 8 & 16 & $8.4 \mathrm{E} 01$ & 3.0 & 2.70 \\
$\mathrm{Si}$ & 14 & 32 & $1.2 \mathrm{E} 01$ & 3.0 & 2.65 \\
$\mathrm{Fe}$ & 26 & 56 & $9.2 \mathrm{E} 00$ & 3.1 & 2.60 \\
\hline
\end{tabular}

where the coefficients $b_{i}=1.2$ and $d_{i}=0.034$ describe the form of the spectrum at low energies $(R<0.55 \mathrm{GV})$ and were inferred from our data on the fluence of oxygen nuclei obtained in the Cosmos2044 experiment (Marenny et al., 1990).

\subsection{Function of muclei penetrating to low orbits}

The GCR particle energy spectra on the satellite orbit were found by calculating the penetrating function on the basis of previous determinations of the boundaries for proton penetration into the magnetosphere in a Cosmos-800 experiment (Biryukov el al., 1984).

Figure I shows the plots of the penetration function, calculated as indicated above and using the conventional techniques based on the International Reference Geomagnetic Field (IRGF) model. Our approach has yielded bigher fluences of particles penetrating to low orbits as compared with conventional techniques (Nymmik, 1991).

\subsection{Radiation belt protons}

The RB proton fluxes on the Cosmos-2044 orbit were calculated using the AP-8 model (Sawyer and Vetter, 1976) for solar maximum. The calculations have shown that the orbit-integrated differential energy spectrum of the $1-100 \mathrm{MeV}$ protons can be described as

$$
F(E)=8 \times 10^{3} E^{-7},
$$

where $y=2.03$ at $E<8 \mathrm{MeV}$ and $\gamma=2.72$ at $E \geqslant 8 \mathrm{MeV}$.

\subsection{Particle flux deep in matter}

The species $i$ particle flux under shielding of thickness $x$ is determined by the formula

$$
F_{x i}\left(E_{x}^{\prime}\right)=F_{\alpha}\left(L_{0} / L_{x}\right)_{i} \exp \left(-x / \lambda_{i}\right)
$$

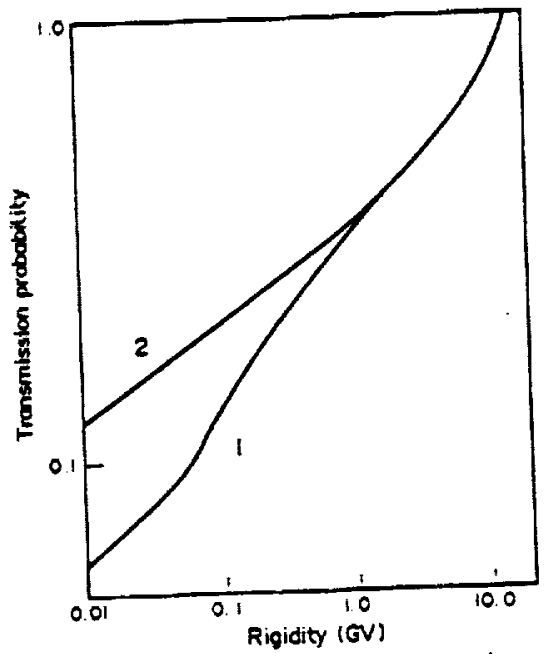

FiG. I. Particle penetration function into the magnetosphere for the Cosmos-2044 orbit. Curve I is the IGRF model calculation result. Curve 2 is the Nymmik (1991) model calculation result. 


\section{CALCULATION AND MEASUREMENTS OF CR IN LEO}

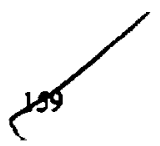

where $E^{r}=E_{0}-\int_{0} L_{1}(x) \mathrm{d} x ; L_{0}$ and $L_{x}$ are the particle LET values above and under the shielding, respectively; $\lambda_{i}$ is the species $i$ particle path for nuclear interaction.

The particle energy spectra under the shielding were used to find the particle LET spectra:

$$
\Phi_{x}(>L)=\sum_{j} \sum_{i} \int_{L}^{\infty} F_{x i j}(E)\left[\frac{\mathrm{d} L_{i}(E)}{\mathrm{d} E}\right]^{-1} \mathrm{~d} L,
$$

where $L_{1}(E)$ is the energy dependence of species $i$ paricle LET value; $j$ is a given flux component (GCR or $\mathbf{R B}$ ).

In conformity with the selection criteria for events in the USF stacks, the flux $\Phi_{x}(>L)$ was broken into four groups (see Fig. 2):

$$
\Phi_{x}(>L)=\sum_{k=1}^{4} \Phi_{x}^{k}(>E) \text {. }
$$

If $x_{1}$ is the level of the upper surface of the upper detector in a pair of detectors, $x_{2}$ is the level of the lower surface of the lower detector in the pair, and $x$ is the level between $x_{1}$ and $x_{2}$, then the pictorial pattern for breaking into four groups of events is

\begin{tabular}{ccccc}
\hline$x$ & 1 & 2 & 3 & 4 \\
& GCR & SR $_{1}$ & SR $_{2}$ & SR $_{3}$ \\
\hline$X_{1}$ & $\otimes$ & $\bigcirc$ & 0 & $\otimes$ \\
$X$ & $\otimes$ & $\otimes$ & $\otimes$ & $\otimes$ \\
$X_{2}$ & $\otimes$ & $Y$ & $\otimes$ & $Y$ \\
\hline
\end{tabular}

where $\otimes$ means that $L>L_{0}$, $\bigcirc$ means that $L<L_{0}$, and $Y$ means that a particle is not present. The

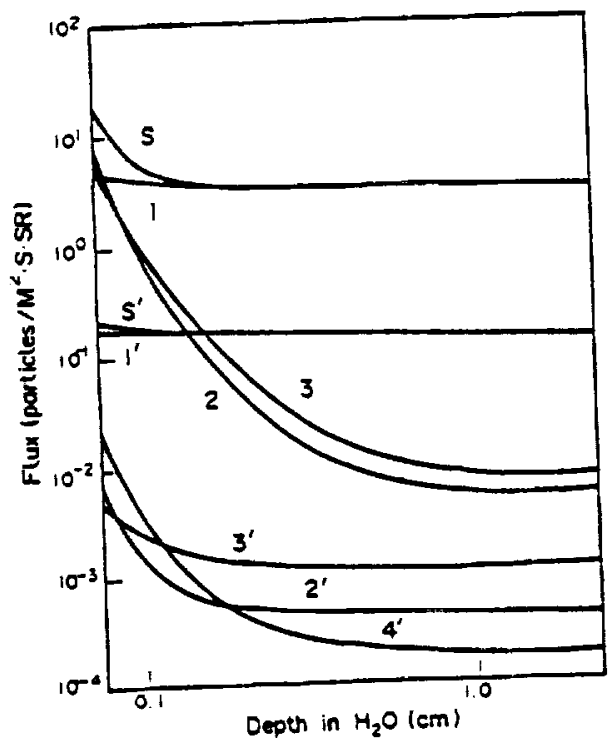

Fic. 2. The caiculated flux vs detector depth. Curves $\mathrm{I}-4$ are particle fluxes calculated by formulas $(8)-(11)$, respectively. $S$ is the total particle flux with $L>L_{0}$ at depth $x$. The curves numbered without a prime are for $L>40 \mathrm{MeV} \mathrm{cm}^{-1}$; those with a prime are for $L>1000 \mathrm{MeV} \mathrm{cm} \mathrm{g}^{-1}$. Curve $4^{\prime}$ is not present for the values of LET and plastic thickness used. particle fluxes at $L>L_{0}$ at the level $x$ may be described by

$$
\Phi_{s}^{1}(G C R)=\sum_{i} \Phi_{i}\left(L \geqslant L_{0}, x_{1}\right) ;\left(E(R)>E\left(x_{2}-x_{1}\right)\right)
$$

$$
\Phi_{x}^{4}(\mathrm{SR})=\sum_{V} \Phi_{j}\left(L \geqslant L_{0}, x_{1}\right) ;\left(E(R) \leqslant E\left(x_{2}-x_{1}\right)\right)
$$

$$
\begin{aligned}
& \Phi_{x}^{2}(\mathrm{SR})=\sum_{i} \Phi_{i j}\left(L<L_{0}, x_{1}\right) ;\left(E(R) \leqslant E\left(x_{2}-x_{1}\right)\right) \\
& \Phi_{x}^{3}(\mathrm{SR})=\sum_{y} \Phi_{4}\left(L<L_{0}, x_{1}\right) ;\left(E(R)>E\left(x_{2}-x_{1}\right)\right) .
\end{aligned}
$$

In the expressions (8)-(11), the GCR group includes the particles with LET values $L>L_{0}$ over a range great enough to penetrate both detector layers, from $x_{1}$ to $x_{2}$.

\section{RESULTS AND DISCUSSION}

Figure 2 shows the $\Phi_{x}^{k}\left(L>L_{0}\right)$, plots $\Phi_{x}^{1}(G C R)$, $\Phi_{x}^{2}(\mathrm{SR}), \Phi_{x}^{3}(\mathrm{SR})$, and $\Phi_{x}^{4}(\mathrm{SR})$ calculated for the experimental conditions under the shielding thicknesses ranging from 0.075 to $2.5 \mathrm{~cm} \mathrm{H}_{2} \mathrm{O}$ at two threshold LET values (40 and $100 \mathrm{MeV} \mathrm{cm} \mathrm{cm}^{-2}$ ). From the calculations it follows that, under the shielding exceeding $0.2 \mathrm{~cm} \mathrm{H}_{2} \mathrm{O}$, the fraction of the events in groups $2-4$ (SR) is less than $1 \%$ of all the detected GCR particles, while the events of group 1 include nearly the total fuence of detected CR particles within the given component. Secondary particles are not included in Fig. 2.

Figure 3 shows the experimental data (plotted points) for (a) the particle fluences detected at seven depths at $x>0.2 \mathrm{~cm} \mathrm{H}, O$ in the USF stacks (GCR) at $L>100$ and $L>500 \mathrm{MeV} \mathrm{cm} \mathrm{g}^{-1}$; and (b) at two depths $(0.24$ and $1.53 \mathrm{~cm})$ in the IMBP stack at $L>1600 \mathrm{MeV} \mathrm{cm} \mathrm{g}^{-1}$. The independence of the values of GCR particle fluxes predicted by calculations for $L>L_{0}$ and at depths of $0.1-2.0 \mathrm{~g} \mathrm{~cm}^{-2}$ is confirmed by the data. The measured particle flux at $L>100 \mathrm{MeV} \mathrm{cm}^{2} \mathrm{~g}^{-1}$ is somewhat below that predicted by the calculations, but probably within the overall accuracy expected from the comparison.

Figure 3 also presents the data on the events selected by the criteria of SR conditions (9)-(11) and shows the calculated dependence which follows from the RB proton spectrum. This data (Curve 1) has been normalized to the SR particle flux at $x=0.25 \mathrm{~cm} \mathrm{H} \mathrm{H}_{2}$. To that end, the factor $C$ in (4) had to be set equal to 1000 , i.e. eight times less than the value given by the AP-8 model for solar maximum. The reduction in proton lux from the model predic. tion can be attributed to uncertainties deriving from 


\section{A. M. MARENNY et al.}

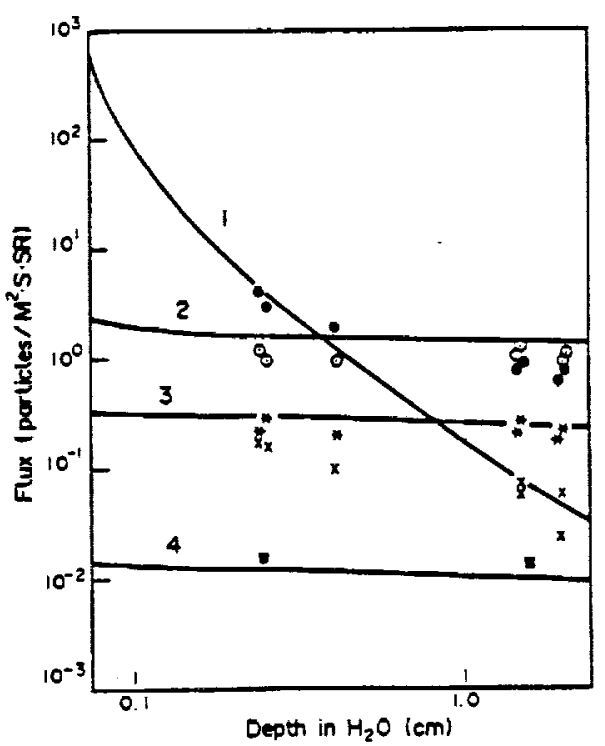

Fig. 3. Particle fluxes with $L>L_{\text {a }}$ vs detector depth. $L>100 \mathrm{meV} \mathrm{cm} \mathrm{g}^{-1}$ : Curve 1 is the $S R$ track density due to RB proton fiux calculated by formula (4); Curve 2 is the track density due to GCR particie flux calculated by formulas (1)-(3); the black circles are experimental SR track densities; the eircles with dots are experimental $G C R$ track densities. $L>500 \mathrm{MeV} \mathrm{cm} g^{-1}$ : Curve 3 is the track density due to GCR particle flux calculated by formulas (1)-(3); the star-like erosses are experimental GCR track densities; the skewed crosses are the SR track densities. I $>2 \times 10^{3} \mathrm{MeV} \mathrm{cm} \mathrm{g}^{-1}$ : Curve 4 is the GCR track density calculated by formulas (1)-(3); the squares are the experimental GCR track densities.

two major causes. There are substantial proton flux fluctuations observed at low altitudes near the lower RB border, where the density of residual atmosphere increases. The high solar activity level during the observations may have contributed to atmospheric expansion and a decrease in proton flux. Also, the pronounced directionality of the trapped proton fluxes is not taken into account by the AP. 8 model or compensated for by the SSNTDs. The assumption of isotropic proton incidence which is made in correcting the experimental measurements for detector solid angle response could therefore have introduced some error into the measured proton fluxes.

From Fig. 3 it follows that the SR particle tlux at $x>0.3 \mathrm{~cm} \mathrm{H} \mathrm{H}_{2} \mathrm{O}$ is considerably greater than would be expected from the primary RB protons. For the particle flux with $L>100 \mathrm{MeV} \mathrm{cm}^{2} \mathrm{~g}^{-1}$, the excess equals about 1 particle $\left(\mathrm{m}^{2} \mathrm{~s} s\right)^{-1}$. Only a small part of this can be attributed to $\mathrm{H}$ recoils produced by neutron collisions. The high energy ( $>1 \mathrm{MeV}$ ) neutron flux was measured on Cosmos-2044 (Dudkin et al., 1992) in the range of $275-500 \mathrm{~m}_{0} / \mathrm{m}^{-2} \mathrm{~s}^{-1} \mathrm{sr}^{-1}$. The efficiency of CR-39 for high energy neutron flux detection has been reported as $\sim 10^{-4}$ proton tracks (neutron) $)^{-1}$ (Durrani and Bull, 1987). This would imply a neutron-induced component of $\sim 0.04$ particles $\mathrm{m}^{-2} \mathrm{~s}^{-1} \mathrm{sr}^{-1}$. High energy protons also contribute through elastic scattering of target $\mathrm{H}$ nuclei and this would seem to be the major source of the excess tracks seen at the larger shielding depths.

The SR particles at $L>500 \mathrm{MeV} \mathrm{cm}^{2} \mathrm{~g}^{-1}$ cannot be protons, but are mainly secondary particles of higher charges produced by inelastic interactions of $\mathrm{RB}$ protons and neutrons with the detector nuclei (H, C, O).

\section{REFERENCES}

Benton E. V. and Parnell T. A. (1988) Space radiation dosimetry on US and Soviet manned missions. In Terrestrial Space Radiation and its Biological Effects, NATO ASI Series A: Life Sciences, Vol. 154, pp. 729-794. Plenum Press, New York.

Biryukov A. S., Ivanova T. A., Kovrigina L. M., Kumetsov S. N., Sosonovets R. N., Tverskaya L. V. and Kudela K. (1984) Boundary of solar cosmic ray penetration into the magnetosphere according to data of Intercosmos. 17 and Cosmos-900. Acto Phys. Slovaca 34, 153.

Dudkin V. E., Potapov Yu. V., Akapova A. B., Melkumyan L. V., Rshtuni Sh. B., Benton E. V. and Frank A. L. (1992) Neutron fluences and energy spectra in the Cosmos-2044 biosatellite orbit. Nucl. Tracks Radiat. Mear. 20, 139-141.

Durrani S. A. and Bull R. K. (1987) Solid State Nuclear Track Delection: Principles, Methods and Applications, International Series in Natural Philosophy, Vol. III, Pergamon Press, Oxford.

Marenny A. M. (1987) Dielectric Nuclear Track Detectors in Space Radiation and Radiobiology Experimenis. Energoatomizdat, Moscow (in Russian).

Marenny A. M., Nymmik R. A., Hunyadi I., Csige I., Spurdy F., Charvat J. and Guertzen G. R. (1990). Low-energy heavy ions of cosmic rays measured on Cosmos-2044 biosatellite. Proc. ISth Int. Conf. Particle Tracks Solids, Marburg, F.R.G., 3-7 September 1990.

Marenny A. M., Nymmik R. A. and Susiov A. A. (1987) Studies of the fluxes of GCR heavy nuclei using the measurements on board low-orbiting satellites in 1974-1984. Kosmich. Issled. 25(4), 577-584.

Nymmik R. A. (1991) Diumal variations of geomagnetic cutoff boundary relevant to penetration function. Kos. mich Issled. 29(3), 491-493.

Sawyer D. M. and Vette J. I. (1976) AP-8 Trapped Prolon Environment for Solar Maximum and Solar Minimum. National Space Science Data Center, Goddard Space Flight Center, NSSDC,WDC-A-R\&S 76-06 (June). Also see NASA TM-X-7260s (1976)

Standards for Galoctic Cosmic Rays: Model for Particle Flux Variations (1991) All-Union State Standard 25645.150190. Standarizdat, Moscow.

Suslov A. A. and Nymmik R. A. (1988) Large-scale galactic cosmic ray modulation: energy (rigidity) spectra of protons and He nuclei outside the modulation region. lev. Akad. Nauk U.S.S.R., Phys. Ser. 58(12), 2330-2334.

Suslov A. A. and Nymmik R.A. (1990) A semi-empirical model for the large-scale modulation of the galactic cosmic ray energy spectra. Proc. 2Isi ICRC 6, 33-37. 